\title{
A classification theorem for hypersurfaces of Minkowski spaces
}

\author{
JINTANG LI
}

\begin{abstract}
Let $M^{n}$ be a compact hypersurface of a Minkowski space $\left(V^{n+1}, \bar{F}\right)$. In this paper, using the Gauss formula of the Chern connection for Finsler submanifolds, we prove that if the second mean curvature $\mathrm{H}_{2}$ of $M$ is constant and the norm square $S$ of the second fundamental form of $M$ satisfies $S \leq \frac{n(n-1)}{n-2} H_{2}$, then $M$ with the induced metric is isometric to the standard Euclidean sphere. This generalizes the result of [2] from the Euclidean to the Minkowski space.
\end{abstract}

Mathematics Subject Classification (2010): 53C60 (primary); 53C40 (secondary).

\section{Introduction}

Let $M$ be an $n$-dimensional smooth manifold and $\pi: T M \rightarrow M$ be the natural projection from the tangent bundle. Let $(x, Y)$ be a point of $T M$ with $x \in M, Y \in$ $T_{x} M$ and let $\left(x^{i}, Y^{i}\right)$ be local coordinates on $T M$ with $Y=Y^{i} \frac{\partial}{\partial x^{i}}$. A Finsler metric on $M$ is a function $F: T M \rightarrow[0,+\infty)$ satisfying the following properties:

(i) Regularity: $F(x, Y)$ is smooth on $T M \backslash 0$;

(ii) Positive homogeneity: $F(x, \lambda Y)=\lambda F(x, Y)$ for $\lambda>0$;

(iii) Strong convexity: The fundamental quadratic form $g_{Y}=g_{i j}(x, Y) d x^{i} \otimes d x^{j}$ is positively definite, where $g_{i j}=\frac{1}{2} \partial^{2}\left(F^{2}\right) / \partial Y^{i} \partial Y^{j}$.

The simplest class of Finsler manifolds is Minkowski space. Let $V^{n+1}$ be a real vector space. A Finsler metric $\bar{F}: T V^{n+1} \rightarrow[0, \infty)$ is called Minkowski if $\bar{F}$ is a function of $\bar{Y} \in V^{n+1}$ only. In this case $\left(V^{n+1}, \bar{F}\right)$ is called a Minkowski space.

Riemannian submanifolds are important in modern differential geometry. There has been a long history for the study of Riemannian submanifolds. For a compact Riemannian hypersurface $M$ of Euclidean space, the second fundamental form is $B=h_{i j}^{n+1} \omega^{i} \otimes \omega^{j} \otimes e_{n+1}$, where $\left\{\omega^{i}\right\}$ is the orthonormal coframe of $M$. The

This research was supported by the Natural Science Foundation of Fujian Province of China (No. 2012J01020).

Received May 27, 2012; accepted December 10, 2012. 
second mean curvature $H_{2}$ of $M$ is defined by $H_{2}=\frac{2}{n(n-1)} \sum_{1 \leq i<j \leq n} \lambda_{i} \lambda_{j}$, where $\lambda_{i}$ are the eigenvalues of the second fundamental tensor $h_{i j}^{n+1}$ of $M$. For a compact hypersurface $M$ of Euclidean space, the Gauss equation is $\sum_{i, j} R_{i j i j}=n(n-1) H_{2}$, which implies that the scalar curvature is constant if and only if the second mean curvature $\mathrm{H}_{2}$ is constant.

As well kown, using Cheng-Yau's self-adjoint operator $\square$, Li [2] proved that if the second mean curvature $\mathrm{H}_{2}$ is constant and the norm square $S$ of the second fundamental form of $M$ satisfies $S \leq \frac{n(n-1)}{n-2} H_{2}$, then $M$ is a Riemannian sphere. As far as we know, there are very few rigidity results on Finsler submanifolds. The main purpose of this paper is to generalize the above result of $\mathrm{Li}$ from the Euclidean to the Minkowski space. In this paper, using the Gauss formula for the Chern connection and defining a similar self-adjoint operator $\square$ on Finsler manifolds, we study the hypersurfaces of Minkowski space $\left(V^{n+1}, \bar{F}\right)$ and we obtain the following:

Main Theorem. Let $M^{n}$ be a compact hypersurface of Minkowski space $\left(V^{n+1}, \bar{F}\right)$. If the second mean curvature $\mathrm{H}_{2}$ is constant and the norm square $\mathrm{S}$ of the second fundamental form of $M$ satisfies $S \leq \frac{n(n-1)}{n-2} H_{2}$, then $M$ with the induced metric is isometric to the standard Euclidean sphere.

\section{Preliminaries}

Let $\left(M^{n}, F\right)$ be an $n$-dimensional Finsler manifold. Then $F$ inherits the Hilbert form, the fundamental tensor and the Cartan tensor as follows [1]:

$$
\begin{aligned}
\omega & =\frac{\partial F}{\partial Y^{i}} d x^{i}, \\
g_{Y} & =g_{i j}(x, Y) d x^{i} \otimes d x^{j}, \\
A_{Y} & =A_{i j k} d x^{i} \otimes d x^{j} \otimes d x^{k}, \\
A_{i j k} & :=\frac{F \partial g_{i j}}{2 \partial Y^{k}} .
\end{aligned}
$$

Let $\varphi:\left(M^{n}, F\right) \rightarrow\left(\bar{M}^{n+p}, \bar{F}\right)$ be an isometric immersion from a Finsler manifold to another one. We have [7]

$$
\begin{gathered}
F(Y)=\bar{F}\left(\varphi_{*}(Y)\right), \quad g_{Y}(U, V)=\bar{g}_{\varphi_{*}(Y)}\left(\varphi_{*}(U), \varphi_{*}(V)\right), \\
A_{Y}(U, V, W)=\bar{A}_{\varphi_{*}(Y)}\left(\varphi_{*}(U), \varphi_{*}(V), \varphi_{*}(W)\right),
\end{gathered}
$$

where $Y, U, V, W \in T M, \bar{g}$ and $\bar{A}$ are the fundamental tensor and the Cartan tensor of $\bar{M}$, respectively. It can be seen from $(2.1)$ that $\varphi^{*}(\bar{\omega})=\omega$, where $\bar{\omega}$ is the Hilbert form of $\bar{M}$.

In the following we simplify $A_{Y}$ and $g_{Y}$ to $A$ and $g$, respectively. Moreover any vector $U \in T M$ will be identified with the corresponding vector $\varphi_{*}(U) \in T \bar{M}$ 
and we will use the following convention:

$1 \leq i, j, \cdots \leq n, n+1 \leq \alpha, \beta, \cdots \leq n+p, 1 \leq \lambda, \mu, \cdots \leq n-1,1 \leq a, b, \cdots \leq n+p$.

Let $\varphi:\left(M^{n}, F\right) \rightarrow\left(\bar{M}^{n+p}, \bar{F}\right)$ be an isometric immersion from a Finsler manifold to another one. Take a $\bar{g}$-orthonormal frame form $\left\{e_{a}\right\}$ for each fibre of $\pi^{*} T \bar{M}$ and let $\left\{\omega^{a}\right\}$ be its local dual coframe, such that $\left\{e_{i}\right\}$ is a frame field for each fibre of $\pi^{*} T M$ and $\omega^{n}$ is the Hilbert form, where $\pi: T M \rightarrow M$ denotes the natural projection. Let $\theta_{b}^{a}$ and $\omega_{j}^{i}$ denote the Chern connection 1-form of $\bar{F}$ and $F$, respectively, i.e. $\bar{\nabla} e_{a}=\theta_{a}^{b} e_{b}$ and $\nabla e_{i}=\omega_{i}^{j} e_{j}$, where $\bar{\nabla}$ and $\nabla$ are the Chern connections of $\bar{M}$ and $M$, respectively. We obtain that $A\left(e_{i}, e_{j}, e_{n}\right)=\bar{A}\left(e_{a}, e_{b}, e_{n}\right)=0$, where $e_{n}=\frac{Y^{i}}{F} \frac{\partial}{\partial x^{i}}$ is the natural dual of the Hilbert form $\omega^{n}$.

The structure equations of $\bar{M}$ are given by

$$
\left\{\begin{array}{l}
d \theta^{a}=-\theta_{b}^{a} \wedge \theta^{b} \\
d \theta_{b}^{a}=-\theta_{c}^{a} \wedge \theta_{b}^{c}+\frac{1}{2} \bar{R}_{b c d}^{a} \omega^{c} \wedge \omega^{d}+\bar{P}_{b c d}^{a} \omega^{c} \wedge \theta_{n}^{d} \\
\theta_{b}^{a}+\theta_{a}^{b}=-2 \bar{A}_{a b c} \theta_{n}^{c} \\
\theta_{n}^{a}+\theta_{a}^{n}=0, \quad \theta_{n}^{n}=0 .
\end{array}\right.
$$

By $\theta^{\alpha}=0$ and the structure equations of $\bar{M}$, we have that $\theta_{j}^{\alpha} \wedge \omega^{j}=0$, which implies that $\theta_{j}^{\alpha}=h_{i j}^{\alpha} \omega^{i}, \quad h_{i j}^{\alpha}=h_{j i}^{\alpha}$. We obtain [3]

$$
\omega_{i}^{j}=\theta_{i}^{j}-\Psi_{j i k} \omega^{k}
$$

where

$$
\begin{aligned}
\Psi_{j i k}= & h_{j n}^{\alpha} \bar{A}_{k i \alpha}-h_{k n}^{\alpha} \bar{A}_{j i \alpha}-h_{i n}^{\alpha} \bar{A}_{k j \alpha}-h_{n n}^{\alpha} \bar{A}_{i k s} \bar{A}_{s j \alpha}+h_{n n}^{\alpha} \bar{A}_{i j s} \bar{A}_{s k \alpha} \\
& +h_{n n}^{\alpha} \bar{A}_{j k s} \bar{A}_{s i \alpha} .
\end{aligned}
$$

In particular,

$$
\omega_{i}^{n}=\theta_{i}^{n}-h_{n n}^{\alpha} \bar{A}_{k i \alpha} \omega^{k} .
$$

Using the almost $\bar{g}$-compatibility, we have

$$
\theta_{\alpha}^{j}=\left(-h_{i j}^{\alpha}-2 h_{n i}^{\beta} \bar{A}_{j \alpha \beta}+2 h_{n n}^{\beta} \bar{A}_{j \lambda \alpha} \bar{A}_{i \lambda \beta}\right) \omega^{i}-2 \bar{A}_{j \alpha \lambda} \omega_{n}^{\lambda} .
$$

In particular, $\theta_{\alpha}^{n}=-h_{n i}^{\alpha} \omega^{i}$. We quote the following results:

Proposition 2.1 ([3]). Let $\varphi:\left(M^{n}, F\right) \rightarrow\left(\bar{M}^{n+p}, \bar{F}\right)$ be an isometric immersion from a Finsler manifold to a Minkowski space. Then

$$
\left\{\begin{array}{l}
\left(\bar{\nabla}_{e_{i}} \bar{A}\right)(\bullet, \bullet, \bullet)=0 \\
\bar{A}\left(\bullet, \bullet, \bar{\nabla}_{e_{i}} e_{n}\right)=0 .
\end{array}\right.
$$


Proposition 2.2 ([3] Gauss equations). Let $\varphi:\left(M^{n}, F\right) \rightarrow\left(\bar{M}^{n+p}, \bar{F}\right)$ be an isometric immersion from a Finsler manifold another one. Then we have

$$
\left\{\begin{aligned}
P_{i k \lambda}^{j}= & \bar{P}_{i k \lambda}^{j}+\Psi_{j i k ; \lambda}-2 \Psi_{s i k} A_{j s \lambda}-2 h_{i k}^{\alpha} \bar{A}_{j \lambda \alpha}, \\
R_{i k l}^{j}= & \bar{R}_{i k l}^{j}-h_{i k}^{\alpha} h_{j l}^{\alpha}+h_{i l}^{\alpha} h_{j k}^{\alpha}+\Psi_{j i k \mid l}-\Psi_{j i l \mid k} \\
& +\Psi_{s i k} \Psi_{j s l}-\Psi_{s i l} \Psi_{j s k}-2 h_{i k}^{\alpha} h_{n l}^{\beta} \bar{A}_{j \alpha \beta}+2 h_{i l}^{\alpha} h_{n k}^{\beta} \bar{A}_{j \alpha \beta} \\
& +2 h_{i k}^{\alpha} h_{n n}^{\beta} \bar{A}_{j s \alpha} \bar{A}_{l s \beta}-2 h_{i l}^{\alpha} h_{n n}^{\beta} \bar{A}_{j s \alpha} \bar{A}_{k s \beta}-h_{n n}^{\alpha} \bar{A}_{s l \alpha} \bar{P}_{i k s}^{j} \\
& +h_{n n}^{\alpha} \bar{A}_{s k \alpha} \bar{P}_{i l s}^{j}+h_{n l}^{\alpha} \bar{P}_{i k \alpha}^{j}-h_{n k}^{\alpha} \bar{P}_{i l \alpha}^{j}
\end{aligned}\right.
$$

where ";" and "|" respectively denote the vertical and the horizontal covariant differentials with respect to the Chern connection $\nabla$.

Proposition 2.3 ([3] Codazzi equations). Let $\varphi:\left(M^{n}, F\right) \rightarrow\left(\bar{M}^{n+p}, \bar{F}\right)$ be an isometric immersion from a Finsler manifold to a Finsler manifold to another one. Then we have

$$
\left\{\begin{array}{l}
h_{i j ; \lambda}^{\alpha}=-\bar{P}_{i j \lambda}^{\alpha}, \\
h_{i j \mid k}^{\alpha}-h_{i k \mid j}^{\alpha}=-\bar{R}_{i j k}^{\alpha}+h_{n j}^{\beta} \bar{P}_{i k \beta}^{\alpha}-h_{n k}^{\beta} \bar{P}_{i j \beta}^{\alpha} \\
-h_{l k}^{\alpha} \Psi_{l i j}+h_{l j}^{\alpha} \Psi_{l i k}-h_{n n}^{\beta} \bar{A}_{l j \beta} \bar{P}_{i k l}^{\alpha}+h_{n n}^{\beta} \bar{A}_{l k \beta} \bar{P}_{i j l}^{\alpha} .
\end{array}\right.
$$

\section{Hypersurfaces of a Minkowski space}

Let $\left(M^{n}, F\right)$ be a compact hypersurfaces of a Minkowski space $\left(V^{n+1}, \bar{F}\right)$. Then we have

$$
h_{i j \mid k}^{n+1} \omega^{k}+h_{i j ; \lambda}^{n+1} \omega_{n}^{\lambda}=d h_{i j}^{n+1}-h_{k j}^{n+1} \omega_{i}^{k}-h_{i k}^{n+1} \omega_{j}^{k}+h_{i j}^{n+1} \theta_{n+1}^{n+1} .
$$

Exterior differentiation of the left-hand side terms of (3.1), gives

$$
\begin{aligned}
d & h_{i j \mid k}^{n+1} \wedge \omega^{k}+h_{i j \mid k}^{n+1} d \omega^{k}+d h_{i j ; \lambda}^{n+1} \wedge \omega_{n}^{\lambda}+h_{i j ; \lambda}^{n+1} d \omega_{n}^{\lambda} \\
= & \left\{h_{i j|k| l}^{n+1} \omega^{l}+h_{i j \mid k ; \mu}^{n+1} \omega_{n}^{\mu}+h_{l j \mid k}^{n+1} \omega_{i}^{l}+h_{i l \mid k}^{n+1} \omega_{j}^{l}+h_{i j \mid l}^{n+1} \omega_{k}^{l}-h_{i j \mid k}^{n+1} \theta_{n+1}^{n+1}\right\} \wedge \omega^{k} \\
& +h_{i j \mid k}^{n+1}\left\{-\omega_{l}^{k} \wedge \omega^{l}\right\} \\
& +\left\{h_{i j ; \lambda \mid l}^{n+1} \omega^{l}+h_{i j ; \lambda ; \mu}^{n+1} \omega_{n}^{\mu}+h_{l j ; \lambda}^{n+1} \omega_{i}^{l}+h_{i l ; \lambda}^{n+1} \omega_{j}^{l}+h_{i j ; \mu}^{n+1} \omega_{\lambda}^{\mu}-h_{i j ; \lambda}^{n+1} \theta_{n+1}^{n+1}\right\} \wedge \omega_{n}^{\lambda} \\
& +h_{i j ; \lambda}^{n+1}\left\{-\omega_{\mu}^{\lambda} \wedge \omega_{n}^{\mu}+\frac{1}{2} R_{n l s}^{\lambda} \omega^{l} \wedge \omega^{s}+P_{n l \mu}^{\lambda} \omega^{l} \wedge \omega_{n}^{\mu}\right\} \\
= & \left\{-h_{i j|k| l}^{n+1}+\frac{1}{2} h_{i j ; \lambda}^{n+1} R_{n k l}^{\lambda}\right\} \omega^{k} \wedge \omega^{l}-h_{i j ; \lambda ; \mu}^{n+1} \omega_{n}^{\lambda} \wedge \omega_{n}^{\mu} \\
& +\left\{-h_{i j \mid k ; \lambda}^{n+1}+h_{i j ; \lambda \mid k}^{n+1}+h_{i j ; \mu}^{n+1} P_{n k l}^{\mu}+h_{i j \mid k}^{n+1} \bar{A}_{n+1 n} n+1 \lambda\right\} \omega^{k} \wedge \omega_{n}^{\lambda} \\
& +h_{l j \mid k}^{n+1} \omega_{i}^{l} \wedge \omega^{k}+h_{i l \mid k}^{n+1} \omega_{j}^{l} \wedge \omega^{k}+h_{l j ; \lambda}^{n+1} \omega_{i}^{l} \wedge \omega_{n}^{\lambda}+h_{i l ; \lambda}^{n+1} \omega_{j}^{l} \wedge \omega_{n}^{\lambda} \\
& -h_{i j \mid k}^{n+1} \theta_{n+1}^{n+1} \wedge \omega^{k}-h_{i j ; \lambda}^{n+1} \theta_{n+1}^{n+1} \wedge \omega_{n}^{\lambda} .
\end{aligned}
$$


Exterior differentiation of the right-hand side terms of (3.1), gives also

$$
\begin{aligned}
& -d h_{k j}^{n+1} \wedge \omega_{i}^{k}-h_{k j}^{n+1} d \omega_{i}^{k}-d h_{i k}^{n+1} \wedge \omega_{j}^{k}-h_{i k}^{n+1} d \omega_{j}^{k} \\
& +d h_{i j}^{n+1} \wedge \theta_{n+1}^{n+1}+h_{i j}^{n+1} d \theta_{n+1}^{n+1} \\
& =-\left\{h_{k j \mid l}^{n+1} \omega^{l}+h_{k j ; \lambda}^{n+1} \omega_{n}^{\lambda}+h_{l j}^{n+1} \omega_{k}^{l}+h_{k l}^{n+1} \omega_{j}^{l}-h_{k j}^{n+1} \theta_{n+1}^{n+1}\right\} \wedge \omega_{i}^{k} \\
& -h_{k j}^{n+1}\left\{-\omega_{l}^{k} \wedge \omega_{i}^{l}+\frac{1}{2} R_{i l s}^{k} \omega^{l} \wedge \omega^{s}+P_{i l \lambda}^{k} \omega^{l} \wedge \omega_{n}^{\lambda}\right\} \\
& -\left\{h_{i k \mid l}^{n+1} \omega^{l}+h_{i k ; \lambda}^{n+1} \omega_{n}^{\lambda}+h_{l k}^{n+1} \omega_{i}^{l}+h_{i l}^{n+1} \omega_{k}^{l}-h_{i k}^{n+1} \theta_{n+1}^{n+1}\right\} \wedge \omega_{j}^{k} \\
& -h_{i k}^{n+1}\left\{-\omega_{l}^{k} \wedge \omega_{j}^{l}+\frac{1}{2} R_{j l s}^{k} \omega^{l} \wedge \omega^{s}+P_{j l \lambda}^{k} \omega^{l} \wedge \omega_{n}^{\lambda}\right\} \\
& +\left\{h_{i j \mid k}^{n+1} \omega^{k}+h_{i j ; \lambda}^{n+1} \omega_{n}^{\lambda}+h_{k j}^{n+1} \omega_{i}^{k}+h_{i k}^{n+1} \omega_{j}^{k}\right\} \wedge \theta_{n+1}^{n+1} \\
& +h_{i j}^{n+1}\left\{2 h_{s k}^{n+1} h_{n l}^{n+1} \bar{A}_{s n+1 n+1}-2 h_{s k}^{n+1} h_{n n}^{n+1} \bar{A}_{s t n+1} \bar{A}_{t l n+1}\right\} \omega^{k} \wedge \omega^{l} \\
& +2 h_{i j}^{n+1} h_{s k}^{n+1} \bar{A}_{s n+1 \lambda} \omega^{k} \wedge \omega_{n}^{\lambda} \\
& =\left\{-\frac{1}{2} h_{s j}^{n+1} R_{i k l}^{s}-\frac{1}{2} h_{i s}^{n+1} R_{j k l}^{k}+2 h_{i j}^{n+1} h_{s k}^{n+1} h_{n l}^{n+1} \bar{A}_{s n+1 n+1}\right. \\
& \left.-2 h_{i j}^{n+1} h_{s k}^{n+1} h_{n n}^{n+1} \bar{A}_{s t n+1} \bar{A}_{t l n+1}\right\} \omega^{k} \wedge \omega^{l} \\
& +\left\{-h_{s j}^{n+1} P_{i k \lambda}^{s}-h_{s j}^{n+1} P_{j k \lambda}^{k}+2 h_{i j}^{n+1} h_{s k}^{n+1} \bar{A}_{s n+1 \lambda}\right\} \omega^{k} \wedge \omega_{n}^{\lambda} \\
& -h_{k j \mid l}^{n+1} \omega^{l} \wedge \omega_{i}^{k}-h_{k j ; \lambda}^{n+1} \omega_{n}^{\lambda} \wedge \omega_{i}^{k}-h_{i k \mid l}^{n+1} \omega^{l} \wedge \omega_{j}^{k}-h_{i k ; \lambda}^{n+1} \omega_{n}^{\lambda} \wedge \omega_{j}^{k} \\
& -h_{i j \mid k}^{n+1} \theta_{n+1}^{n+1} \wedge \omega^{k}-h_{i j ; \lambda}^{n+1} \theta_{n+1}^{n+1} \wedge \omega_{n}^{\lambda} .
\end{aligned}
$$

It can be seen from (3.2) and (3.3) that

$$
\begin{aligned}
& \left\{h_{i j|k| l}^{n+1}-\frac{1}{2} h_{s j}^{n+1} R_{i k l}^{s}-\frac{1}{2} h_{i s}^{n+1} R_{j k l}^{s}-\frac{1}{2} h_{i j ; \lambda}^{n+1} R_{n k l}^{\lambda}\right. \\
& \left.\quad+2 h_{i j}^{n+1} h_{s k}^{n+1} h_{n l}^{n+1} \bar{A}_{s n+1 n+1}-2 h_{i j}^{n+1} h_{s k}^{n+1} h_{n n}^{n+1} \bar{A}_{s t n+1} \bar{A}_{t l n+1}\right\} \omega^{k} \wedge \omega^{l} \\
& +\left\{h_{i j \mid k ; \lambda}^{n+1}-h_{i j ; \lambda \mid k}^{n+1}+h_{i j ; \mu}^{n+1} P_{n k \lambda}^{\mu}+h_{i s}^{n+1} P_{j k l}^{s}+h_{s j}^{n+1} P_{i k l}^{s}-h_{i j \mid k}^{n+1} \bar{A}_{n+1 n+1 \lambda}\right. \\
& \left.\quad+2 h_{i j}^{n+1} h_{s k}^{n+1} \bar{A}_{s n+1 \lambda}\right\} \omega^{k} \wedge \omega_{n}^{\lambda}+h_{i j ; \lambda ; \mu}^{n+1} \omega_{n}^{\lambda} \wedge \omega_{n}^{\mu} \\
& =0 .
\end{aligned}
$$


From (3.4) we immediately obtain the following:

Proposition 3.1. If $M^{n}$ be a hypersurface of Minkowski space $\left(V^{n+1}, \bar{F}\right)$, then

$$
\left\{\begin{aligned}
h_{i j ; \lambda ; \mu}^{n+1}-h_{i j ; \mu ; \lambda}^{n+1}= & 0, \\
h_{i j \mid k ; \lambda}^{n+1}-h_{i j ; \lambda \mid k}^{n+1}= & -h_{s j}^{n+1} P_{i k l}^{s}-h_{i s}^{n+1} P_{j k l}^{s}-h_{i j ; \mu}^{n+1} P_{n k \lambda}^{\mu}+h_{i j \mid k}^{n+1} \bar{A}_{n+1 n+1 \lambda} \\
& -2 h_{i j}^{n+1} h_{s k}^{n+1} \bar{A}_{s n+1 \lambda}, \\
h_{i j|k| l}^{n+1}-h_{i j|l| k}^{n+1}= & h_{s j}^{n+1} R_{i k l}^{s}+h_{i s}^{n+1} R_{j k l}^{s}+h_{i j ; \lambda}^{n+1} R_{n k l}^{\lambda} \\
& -2 h_{i j}^{n+1} h_{s k}^{n+1} h_{n l}^{n+1} \bar{A}_{s n+1 n+1}+2 h_{i j}^{n+1} h_{s l}^{n+1} h_{n k}^{n+1} \bar{A}_{s n+1 n+1} \\
& +2 h_{i j}^{n+1} h_{s k}^{n+1} h_{n n}^{n+1} \bar{A}_{s t n+1} \bar{A}_{t l n+1} \\
& -2 h_{i j}^{n+1} h_{s l}^{n+1} h_{n n}^{n+1} \bar{A}_{s t n+1} \bar{A}_{t k n+1} .
\end{aligned}\right.
$$

The form $B=h_{i j}^{n+1} \omega^{i} \otimes \omega^{j} \otimes e_{n+1}$ is called the second fundamental form of $M$ and $H=\frac{1}{n} \operatorname{tr} B=\frac{1}{n} \sum_{i} h_{i i}^{n+1} e_{n+1}$ is called the mean curvature vector. The norm square $S$ of the second fundamental form of $M$ is $S=\sum_{i j}\left(h_{i j}^{n+1}\right)^{2}$. Let $\lambda_{i}$ be the eigenvalues of the second fundamental tensor $h_{i j}^{n+1}$ of $M$. The second mean curvature $H_{2}=\frac{2}{n(n-1)} \sum_{1 \leq i<j \leq n} \lambda_{i} \lambda_{j}=\frac{1}{n(n-1)}\left[(n H)^{2}-S\right]$.

From the second formula of Proposition 2.1, we obtain that

$$
\bar{A}\left(\bullet, \bullet, \nabla_{e_{i}} e_{n}\right)+\bar{A}\left(\bullet, \bullet, e_{\lambda}\right) \Psi_{\lambda n i}+\bar{A}\left(\bullet, \bullet, e_{n+1}\right) h_{n i}^{n+1}=0 .
$$

Let $P$ be an arbitrary point in $M$. There exists a local coordinate system $\left\{x^{i}\right\}$ such that $\frac{Y^{n}}{F} \frac{\partial}{\partial x^{n}}=e_{n}$. Let $\gamma(t)$ be a curve in $M$ with $\gamma(0)=P$ and tangent vector field $\dot{\gamma}(t)=e_{n}$. Let $X_{i}(t)$ be parallel vector fields along $\gamma(t)$ with $X_{i}(0)=\left.\frac{\partial}{\partial x^{i}}\right|_{p}$. We have that $\nabla_{e_{n}} \frac{\partial}{\partial x^{i}}=\frac{Y^{n}}{F} \nabla_{\frac{\partial}{\partial x^{n}}} \frac{\partial}{\partial x^{i}}=0$ at $P$, i.e. $\Gamma_{n i}^{k}=0$ at $P$, then we can obtain that at $P$

$$
\begin{aligned}
\bar{A}\left(\bullet, \bullet, \nabla_{e_{i}} e_{n}\right) & =\bar{A}\left(\bullet, \bullet, \frac{Y^{n}}{F} \nabla_{u_{i}^{j} \frac{\partial}{\partial x^{j}}} \frac{\partial}{\partial x^{n}}\right) \\
& =\bar{A}\left(\bullet, \bullet, \frac{Y^{n}}{F} u_{i}^{j} \Gamma_{j n}^{k} \frac{\partial}{\partial x^{k}}\right)=0 .
\end{aligned}
$$

Substituting (3.7) into (3.6) yields that $\bar{A}\left(\bullet, \bullet, e_{j}\right) \Psi_{j n i}+\bar{A}\left(\bullet, \bullet, e_{n+1}\right) h_{n i}^{n+1}=0$ at $P$, which together with (2.4) yields that $-h_{n n}^{n+1} \bar{A}_{j i n+1} \bar{A}_{s t j}+h_{n i}^{n+1} \bar{A}_{s t n+1}=0$ at $P$, so by $(2.4)$ we have that

$$
\Psi_{i j k}=0 \text { and } h_{n i}^{n+1} \bar{A}\left(\bullet, \bullet, e_{n+1}\right)=0, \quad \forall i, j, k, \text { at } P .
$$

It follows from the first formula of Proposition 2.1 and $\bar{A}(\bullet, \bullet, \bullet)_{\mid i}=0$ that

$$
\bar{A}(\bullet, \bullet, \bullet)_{; \lambda} \Psi_{\lambda n i}+\bar{A}(\bullet, \bullet, \bullet)_{; n+1} h_{n i}^{n+1}=0 \text { at } P .
$$

It can be seen from (3.8) and (3.9) that

$$
\bar{A}(\bullet, \bullet, \bullet)_{; n+1} h_{n i}^{n+1}=0 \text { at } P .
$$


Now taking the exterior differentiation of $A_{i j k}=\bar{A}_{i j k}$, we obtain that

$$
\begin{aligned}
A_{i j k \mid l}= & \bar{A}_{i j k ; \lambda} \Psi_{\lambda n l}+\bar{A}_{i j k ; n+1} h_{n l}^{n+1} \\
& +\bar{A}_{s j k} \Psi_{s i l}+\bar{A}_{i s k} \Psi_{s j l}+\bar{A}_{i j s} \Psi_{s k l} \\
& +\bar{A}_{n+1 j k} h_{i l}^{n+1}+\bar{A}_{i n+1 k} h_{j l}^{n+1}+\bar{A}_{i j n+1} h_{k l}^{n+1} .
\end{aligned}
$$

By (3.8) and (3.11), we obtain that

$$
A_{i j k \mid n}=0 \text { at } P \text {. }
$$

Define $\delta Y^{i}=d Y^{i}+N_{j}^{i} d x^{j}$. The pull-back of the Sasaki metric $g_{i j} d x^{i} \otimes d x^{j}+$ $g_{i j} \delta Y^{i} \otimes \delta Y^{j}$ from $T M \backslash\{0\}$ to the sphere bundle $S M$ is a Riemannian metric $\widehat{g}=$ $g_{i j} d x^{i} \otimes d x^{j}+\delta_{a b} \omega_{n}^{a} \otimes \omega_{n}^{b}$.

We quote the following results:

Lemma 3.2 ([5]). For $X=\sum_{i} x_{i} \omega^{i} \in \Gamma\left(\pi^{*} T^{*} M\right), \operatorname{div} \widehat{g} X=\sum_{i} x_{i \mid i}+\sum_{\mu, \lambda} x_{\mu} P_{\lambda \lambda \mu}^{n}$. Lemma 3.3 ([6]). Let $B$ be a real symmetric matrix with $\operatorname{tr} B=0$. Then

$$
\left|\operatorname{tr} B^{3}\right| \leq \frac{n-2}{\sqrt{n(n-1)}}\left(\operatorname{tr} B^{2}\right)^{\frac{3}{2}} .
$$

Lemma 3.4 ([4]). All Landsberg spaces of nonzero constant flag curvature must be Riemannian.

Let $\phi=\sum_{i, j} \phi_{i j} \omega^{i} \otimes \omega^{j}$ be a symmetric tensor defined on the sphere bundle $S M$ and $\psi=\sum_{i} \psi_{i} \omega^{i} \in \Gamma\left(\pi^{*} T^{*} M\right)$. Now we can define an operator $\square$ associated to $\phi$ by

$$
\square f=\sum_{i, j} \phi_{i j} f_{|i| j}+\sum_{i, \lambda, \mu} \phi_{i \lambda} f_{\mid i} P_{\mu \mu \lambda}^{n}+\sum_{i} \psi_{i} f_{\mid i}, \quad \forall f \in C^{\infty}(S M) .
$$

Proposition 3.5. Let $(M, F)$ be a compact manifold. Then the operator $\square$ is selfadjoint if and only if $\sum_{j} \phi_{i j \mid j}-\psi_{i}=0$.

Proof. Let $X=\sum_{i, j} g \phi_{i j} f_{\mid i} \omega^{j} \in \Gamma\left(\pi^{*} T^{*} M\right), \quad \forall f, g \in C^{\infty}(S M)$. Then we have from Lemma 3.2

$$
\begin{aligned}
\operatorname{div}_{\widehat{g}} X & =\sum_{i, j}\left\{g_{\mid j} \phi_{i j} f_{\mid i}+g \phi_{i j \mid j} f_{\mid i}+g \phi_{i j} f_{|i| j}\right\}+\sum_{i, \lambda, \mu} g \phi_{i \lambda} f_{\mid i} P_{\mu \mu \lambda}^{n} \\
& =\sum_{i, j} g_{\mid j} \phi_{i j} f_{\mid i}+\sum_{i} g \psi_{i} f_{\mid i}+\sum_{i, j} g \phi_{i j} f_{|i| j}+\sum_{i, \lambda, \mu} g \phi_{i \lambda} f_{\mid i} P_{\mu \mu \lambda}^{n} .
\end{aligned}
$$

Integrating (3.14) yields

$$
\int_{S M}(\square f) g d V_{S M}=-\int_{S M} \sum_{i, j} g_{\mid j} \phi_{i j} f_{\mid i} d V_{S M}
$$

Hence we get

$$
\int_{S M}(\square f) g d V_{S M}=\int_{S M}(\square g) f d V_{S M},
$$

which completes the proof. 


\section{Proof of the main theorem}

Substituting (3.8) into the second formula of Proposition 2.2, by Proposition 2.3 and Proposition 3.1, we obtain that at $P$

$$
\left\{\begin{array}{l}
h_{i j|k| l}^{n+1}=h_{i k|j| l}^{n+1}+h_{s k}^{n+1} \Psi_{s i j \mid l}+h_{s j}^{n+1} \Psi_{s i k \mid l} \\
h_{i j|k| l}^{n+1}=h_{i j|l| k}^{n+1}+h_{s j}^{n+1} R_{i k l}^{s}+h_{i s}^{n+1} R_{j k l}^{s} \\
R_{i k l}^{j}=-h_{i k}^{\alpha} h_{j l}^{\alpha}+h_{i l}^{\alpha} h_{j k}^{\alpha}+\Psi_{j i k \mid l}-\Psi_{j i l \mid k} .
\end{array}\right.
$$

Let $\omega=d S=S_{\mid i} \omega^{i}+S_{; i} \omega_{n}^{i}$. Then $\omega$ is a global section of $\pi^{*} T^{*} M$. By the first formula of (2.24), we have $S_{; i}=0$, i.e. $\omega=d S=S_{\mid i} \omega^{i}$. In the following, the computation is pointwisely estimated. Using the first formula of (4.1) and Lemma 3.2 , we have that

$$
\begin{aligned}
\operatorname{div} \widehat{g} \omega= & 2\left[\sum_{i, j, k} h_{i j}^{n+1} h_{i j \mid k}^{n+1}\right]_{\mid k}+2 \sum_{i, j, k} h_{i j}^{n+1} h_{i j \mid \lambda}^{n+1} P_{\mu \mu \lambda}^{n} \\
= & 2 \sum_{i, j, k}\left(h_{i j \mid k}^{n+1}\right)^{2}+2 \sum_{i, j, k} h_{i j}^{n+1} h_{i k|j| k}^{n+1}+2 \sum_{i, j, k, s} h_{i j}^{n+1} h_{s k}^{n+1} \Psi_{s i j \mid k} \\
& +2 \sum_{i, j, k, s} h_{i j}^{n+1} h_{s j}^{n+1} \Psi_{s i k \mid k}+2 \sum_{i, j, k} h_{i j}^{n+1} h_{i j \mid \lambda}^{n+1} P_{\mu \mu \lambda}^{n} .
\end{aligned}
$$

It can be seen from (4.1) and (4.2) that

$$
\begin{aligned}
\operatorname{div} \widehat{g} \omega= & 2 \sum_{i, j, k}\left(h_{i j \mid k}^{n+1}\right)^{2}+2 \sum_{i, j, k, s} h_{i j}^{n+1}\left\{h_{k i|k| j}^{n+1}+h_{s i}^{n+1} R_{k j k}^{s}+h_{k s}^{n+1} R_{i j k}^{s}\right\} \\
& +2 \sum_{i, j, k, s} h_{i j}^{n+1} h_{s k}^{n+1} \Psi_{s i j \mid k}+2 \sum_{i, j, k, s} h_{i j}^{n+1} h_{s j}^{n+1} \Psi_{s i k \mid k}+2 \sum_{i, j, k} h_{i j}^{n+1} h_{i j \mid \lambda}^{n+1} P_{\mu \mu \lambda}^{n} \\
= & \sum_{i, j, k}\left(h_{i j \mid k}^{n+1}\right)^{2}+2 \sum_{i, j, k, s} h_{i j}^{n+1} h_{k k|i| j}^{n+1}+2 \sum_{i, j, k} h_{i j}^{n+1} h_{i j \mid \lambda}^{n+1} P_{\mu \mu \lambda}^{n} \\
& +n H \sum_{i}\left(\lambda_{i}-H\right)^{3}+3 n H^{2} S-2 n^{2} H^{4}-S^{2} \\
\geq & \sum_{i, j, k}\left(h_{i j \mid k}^{n+1}\right)^{2}+\sum_{i, j, k, s} h_{i j}^{n+1} h_{k k|i| j}^{n+1}+\sum_{i, j, k} h_{i j}^{n+1} h_{i j \mid \lambda}^{n+1} P_{\mu \mu \lambda}^{n} \\
& +\frac{n-1}{n}\left[S-n H_{2}\right]\left\{2(n-1) H_{2}-\frac{n-2}{n} S\right. \\
& \left.-\frac{n-2}{n} \sqrt{\left(n(n-1) H_{2}+S\right)\left(S-n H_{2}\right)}\right\},
\end{aligned}
$$


where the second mean curvature $H_{2}=\frac{1}{n(n-1)}\left[(n H)^{2}-S\right]$. Let

$$
\begin{aligned}
\square f= & \sum_{i, j}\left(n H \delta_{i j}-h_{i j}^{n+1}\right) f_{|i| j}+\sum_{i, \lambda, \mu}\left(n H \delta_{i \lambda}-h_{i \lambda}^{n+1}\right) f_{\mid i} P_{\mu \mu \lambda}^{n} \\
& +\sum_{i, j, k}\left(h_{k j}^{n+1} \Psi_{k j i}-h_{k i}^{n+1} \Psi_{k j j}\right) f_{\mid i} .
\end{aligned}
$$

By the second formula of Proposition 2.3, we can obtain that

$$
\sum_{j}\left(n H \delta_{i j}-h_{i j}^{n+1}\right)_{\mid j}-\sum_{j, k}\left(h_{k j}^{n+1} \Psi_{k j i}-h_{k i}^{n+1} \Psi_{k j j}\right)=0,
$$

which together with Proposition 3.5 implies that the operator $\square$ is self-adjoint. When $\mathrm{H}_{2}$ is constant, we have the following computation by (4.3)

$$
\begin{aligned}
\square(n H)= & \sum_{i} n H(n H)_{|i| i}-\sum_{i, j, k} h_{i j}^{n+1} h_{k k|i| j}^{n+1} \\
& +\sum_{i, \lambda, \mu}\left(n H \delta_{i \lambda}-h_{i \lambda}^{n+1}\right)(n H)_{\mid i} P_{\mu \mu \lambda}^{n} \\
& +\sum_{i, j, k}\left(h_{k j}^{n+1} \Psi_{k j i}-h_{k i}^{n+1} \Psi_{k j j}\right)(n H)_{\mid i} \\
\geq & \sum_{i, j, k}\left(h_{i j \mid k}^{n+1}\right)^{2}-\sum_{i}\left(n H_{\mid i}\right)^{2} \\
& +\frac{(n-1)}{n}\left[S-n H_{2}\right]\left\{2(n-1) H_{2}-\frac{n-2}{n} S\right. \\
& +\sum_{i, \lambda, \mu}\left(n H \delta_{i \lambda}-h_{i \lambda}^{n+1}\right)(n H)_{\mid i} P_{\mu \mu \lambda}^{n} \\
& +\sum_{i, j, k}\left(h_{k j}^{n+1} \Psi_{k j i}-h_{k i}^{n+1} \Psi_{k j j}\right)(n H)_{\mid i} .
\end{aligned}
$$

Using the fact that $P_{i j \lambda}^{n}=-A_{i j \lambda \mid n}$, by (3.12) and (3.8) we have

$$
\begin{aligned}
\square(n H) \geq \sum_{i, j, k}\left(h_{i j \mid k}^{n+1}\right)^{2}-\sum_{i}\left(n H_{\mid i}\right)^{2} \\
+\frac{(n-1)}{n}\left[S-n H_{2}\right]\left\{2(n-1) H_{2}-\frac{n-2}{n} S\right. \\
\left.\quad-\frac{n-2}{n} \sqrt{\left(n(n-1) H_{2}+S\right)\left(S-n H_{2}\right)}\right\} .
\end{aligned}
$$

On the other hand, let $x=\bar{x}^{a} \frac{\partial}{\partial \bar{x}^{a}}$ be the position vector field of the Minkowski space $V^{n+1}$ with respect to the origin. By a direct simple computation, we get 
$\bar{\nabla}_{Z} x=Z, \quad \forall Z=z^{a} \frac{\partial}{\partial \bar{x}^{a}}$ on $V^{n+1}$. This together with the second formula of Proposition 2.1 implies that $\nabla_{e_{i}} x^{2}=2\left\langle e_{i}, x\right\rangle$ and $\nabla_{e_{i}}\left\langle e_{i}, x\right\rangle=\theta_{i}^{j}\left(e_{i}\right)\left\langle e_{j}, x\right\rangle+$ $h_{i i}^{n+1}\left\langle e_{n+1}, x\right\rangle+1$. Then, when $M$ is compact, there exists a point $P \in M$ such that $h_{i i}^{n+1}(P)>0, \forall i$, hence we have that $n(n-1) H_{2}(P)=\sum_{1 \leq i<j \leq n} \lambda_{i}(P) \lambda_{j}(P)>$ 0 , thus the constant $H_{2}>0$, which yields $(n H)^{2}>S$. On the other hand, we have

$$
\sum_{i}(n H)^{2}\left(n H_{\mid i}\right)^{2}=\sum_{i}\left[\sum_{j, k} h_{j k}^{n+1} h_{j k \mid i}^{n+1}\right]^{2} \leq S \sum_{i, j, k}\left[h_{j k \mid i}^{n+1}\right]^{2} .
$$

Hence

$$
\left(n H_{\mid i}\right)^{2} \leq\left(h_{j k \mid i}^{n+1}\right)^{2} .
$$

It can be seen that our assumption $S \leq \frac{n(n-1)}{n-2} H_{2}$ is equivalent to

$$
2(n-1) H_{2}-\frac{n-2}{n} S-\frac{n-2}{n} \sqrt{\left(n(n-1) H_{2}+S\right)\left(S-n H_{2}\right)} \geq 0,
$$

therefore the right-hand side of (4.5) is non-negative by (4.7) and (4.8). Because of the compactness of $M$, we get that $h_{i j}^{n+1}$ is constant and $h_{i j}^{n+1}=0, \forall i \neq j$ on $M$. Exterior differentiation of $h_{n a}^{n+1}=0$ yields $h_{a a}^{n+1}=h_{n n}^{n+1}, \quad \forall a=1, \cdots, n-1$, i.e., $h_{i i}^{n+1}=H, \forall i$. Since $h_{i j \mid k}^{n+1}=0$, it can be seen from the second formula of Proposition 2.3 that

$$
h_{l k}^{n+1} \Psi_{l i j}-h_{l j}^{n+1} \Psi_{l i k}=0 .
$$

Set $j=n, k=\lambda$ in (4.9); by $h_{i j}^{n+1}=0, \forall i \neq j$ we obtain that

$$
h_{\lambda \lambda}^{n+1} \Psi_{\lambda i n}-h_{n n}^{n+1} \Psi_{n i \lambda}=0,
$$

which together with (2.4) yields

$$
h_{n n}^{n+1} \bar{A}_{i j n+1}=0, \forall i, j .
$$

So we get that $\Psi_{i j k}=0$ on $M$. Using the first formula of Proposition 2.2, we have that $P_{i j \lambda}^{n}=0$ on $M$, thus $M$ is a Landsberg space. It is easy to see from the second formula of Proposition 2.2 and $h_{i j}^{n+1}=H \delta_{i j}$ that

$$
R_{i k l}^{j}=H\left(\delta_{i l} \delta_{j k}-\delta_{i k} \delta_{j l}\right) .
$$

It can be seen from $h_{i i}^{n+1}=H$ and the constant $H_{2}=\frac{1}{n(n-1)}\left[(n H)^{2}-S\right]>0$ that $H \neq 0$ is constant, hence we get that $M$ is a Landsberg space with the nonzero constant flag curvature $H$, which together with Lemma 3.4 finishes the proof of Theorem 4.2. 


\section{References}

[1] D.BAO, S. S.CHERN and Z.ShEN, "An Introduction to Riemann-Finsler Geometry", Springer-Verlag, 2000.

[2] H. Li, hypersurfaces with constant scalar curvature in space forms, Math. Ann. 305 (1996), 665-672.

[3] J. T. LI, The pinching theorem for hypersurfaces of Minkowski spaces, J. Math. Anal. Appl. 388 (2012), 1285-1291.

[4] S.NumATA, On Landsberg spaces of scalar curvature, J. Korean Math. Soc. 12 (1975), 97-100.

[5] X. H. Mo, Harmonic maps from Finsler manifolds, Illinois J. Math. 45 (2001), 1331-1345.

[6] W. S ANTOS, Submanifolds with parallel mean curvature vector in spheres, Tohoku Math.J. 46 (1994), 403-415.

[7] Z. SHEN, On Finsler geometry of submanifolds, Math. Ann. 311(1998), 549-576.

School of Mathematical Sciences

Xiamen University

361005 Xiamen, Fujian, China

dli66@xmu.edu.cn 